\title{
The Generalized Green's Function for Boundary Value Problem of Second Order Difference Equation
}

\author{
Xiaoling Han and Juanjuan Huang \\ Department of Mathematics, Northwest Normal University, Lanzhou 730070, China \\ Correspondence should be addressed to Juanjuan Huang; hanxiaoling9@163.com
}

Received 30 June 2014; Accepted 27 July 2014

Academic Editor: Kishin Sadarangani

Copyright (C) 2015 X. Han and J. Huang. This is an open access article distributed under the Creative Commons Attribution License, which permits unrestricted use, distribution, and reproduction in any medium, provided the original work is properly cited.

Let $b>a+2$ and $[a+1, b+1]=\{a+1, a+2, \ldots, b+1\}$. In this paper, by building the generalized Green's function for the problems, we study the solvability of the S-L problem $L x=\Delta[p(t-1) \Delta x(t-1)]+[q(t)+\lambda r(t)] x(t)=-f(t), U_{1}(x)=\alpha_{1} x(a)+\alpha_{2} \Delta x(a)=0$, $U_{2}(x)=\beta_{1} x(b+1)+\beta_{2} \Delta x(b+1)=0$, and the periodic S-L problem $L x=\Delta[p(t-1) \Delta x(t-1)]+[q(t)+\lambda r(t)] x(t)=-f(t)$, $U_{3}(x)=x(a)-x(b+1)=0, U_{4}(x)=\Delta x(a)-\Delta x(b+1)=0$, where the parameter $\lambda$ is an eigenvalue of the linear problem $L x=0$, $U_{1}(x)=0, U_{2}(x)=0$ or the problem $L x=0, U_{3}(x)=0, U_{4}(x)=0$, and $p:[a, b+1] \rightarrow(0,+\infty), r:[a+1, b+1] \rightarrow(0,+\infty), q(t)$ is defined and real valued on $[a+1, b+1], \alpha_{1}^{2}+\alpha_{2}^{2} \neq 0, \beta_{1}^{2}+\beta_{2}^{2} \neq 0$, and in the periodic S-L problem we have $p(a)=p(b+1)$.

\section{Introduction}

There are many results about the solvability of S-L problem

$$
\begin{gathered}
L x=\Delta[p(t-1) \Delta x(t-1)]+[q(t)+\lambda r(t)] x(t)=-f(t), \\
U_{1}(x)=\alpha_{1} x(a)+\alpha_{2} \Delta x(a)=0, \\
U_{2}(x)=\beta_{1} x(b+1)+\beta_{2} \Delta x(b+1)=0
\end{gathered}
$$

and the periodic S-L problem

$$
\begin{gathered}
L x=\Delta[p(t-1) \Delta x(t-1)]+[q(t)+\lambda r(t)] x(t)=-f(t), \\
U_{3}(x)=x(a)-x(b+1)=0, \\
U_{4}(x)=\Delta x(a)-\Delta x(b+1)=0,
\end{gathered}
$$

where $p:[a, b+1] \rightarrow(0,+\infty), r:[a+1, b+1] \rightarrow(0,+\infty)$, and $q(t)$ is defined and real valued on $[a+1, b+1]$, and $[a+$ $1, b+1]=\{a+1, a+2, \ldots, b+1\}$. Consider $\alpha_{1}^{2}+\alpha_{2}^{2} \neq 0, \beta_{1}^{2}+\beta_{2}^{2} \neq$ 0 , in the periodic S-L problem we have $p(a)=p(b+1)$.

If the parameter $\lambda$ is not an eigenvalue of the linear problem

$$
L x=0, \quad U_{1}(x)=0, \quad U_{2}(x)=0
$$

or the periodic S-L problem

$$
L x=0, \quad U_{3}(x)=0, \quad U_{4}(x)=0 ;
$$

then the linear operator is reversible; we can get an equivalent summation form for the problem (1) or problem (2) by using Green's function; there are many results in this case; we refer readers to [1-4]. However, when $\lambda$ is an eigenvalue, we cannot get the summation form in usual way. So, in this paper we introduce the concept of generalized Green's function and construct the generalized Green function for the two problems. On this base we still can convert the originally irreversible difference operator into summation form. Moreover, we also obtain the necessary and sufficient condition for the solvability of problems (1) and (2). We refer readers to [5-10] for more details about discrete equation.

\section{Preliminaries}

We first wish to collect some basic lemmas that will be important to us in what follows.

Definition 1 (see [5]). Let $y(t)$ and $z(t)$ be solutions of $L x=0$; then

$$
\omega[y(t), z(t)]=y(t) \Delta z(t)-z(t) \Delta y(t) .
$$


Lemma 2 (see [5]). If $y(t)$ and $z(t)$ are defined on $[a, b+2]$, then

$$
\begin{aligned}
z(t) & L y(t)-y(t) L z(t) \\
& =\Delta\{p(t-1) \omega[z(t-1), y(t-1)]\}
\end{aligned}
$$

for $t \in[a+1, b+1]$.

By summing both sides of (6) from $a+1$ to $b+1$, we get the following lemma.

Lemma 3 (see [5]). Assume that $y(t)$ and $z(t)$ are defined on $[a, b+2]$. Then

$$
\begin{gathered}
\sum_{t=a+1}^{b+1} z(t) L y(t)-\sum_{t=a+1}^{b+1} y(t) L z(t) \\
=\{p(t) \omega[z(t), y(t)]\}_{a}^{b+1} .
\end{gathered}
$$

Lemma 4 (see [5]). If $y(t)$ and $z(t)$ are solutions of $L x=0$, then $\omega[y(t), z(t)]=C / p(t), t \in[a, b+1]$, where $C$ is $a$ constant.

Remark 5. If $y(t)$ and $z(t)$ are linearly dependent, then $\omega[y(t), z(t)]=0$; if they are linearly independent, then $\omega[y(t), z(t)] \neq 0$.

\section{The Main Results for Problem (1)}

Theorem 6. The semihomogeneous boundary value problem (1) has a solution if and only if

$$
\sum_{\tau=a+1}^{b+1} f(\tau) x_{n}(\tau)=0, \quad n=0,1,2, \ldots
$$

where $x_{n}(t)$ is an eigenfunction corresponding to the eigenvalue $\lambda$.

Proof. Let $x(t)$ be the solution of problem (1) and $x_{n}(t)$ the nontrivial solution for homogeneous problem (3); using

$$
\begin{aligned}
x(t) & L x_{n}(t)-x_{n}(t) \operatorname{Lx}(t) \\
= & \Delta\left\{p(t-1) \omega\left[x(t-1), x_{n}(t-1)\right]\right\}
\end{aligned}
$$

and $L x=-f(t), L x_{n}=0$, we have

$$
\begin{aligned}
\sum_{\tau=a+1}^{b+1} f(\tau) x_{n}(\tau) \\
=\left\{p(t) \omega\left[x(t), x_{n}(t)\right]\right\}_{a}^{b+1} \\
=p(b+1)\left[x(b+1) \Delta x_{n}(b+1)-x_{n}(b+1) \Delta x(b+1)\right] \\
\quad-p(a)\left[x(a) \Delta x_{n}(a)-x_{n}(a) \Delta x(a)\right] .
\end{aligned}
$$

Since $x(t)$ and $x_{n}(t)$ satisfy the boundary condition of (1), then

$$
\sum_{\tau=a+1}^{b+1} f(\tau) x_{n}(\tau)=0
$$

On the other hand, choose a solution $x(t)$ for $L x=-f(t)$, satisfying $x(b+1)=x_{n}(b+1), \Delta x(b+1)=\Delta x_{n}(b+1)$; then

$$
U_{2}(x)=U_{2}\left(x_{n}\right)=\beta_{1} x(b+1)+\beta_{2} \Delta x(b+1)=0 .
$$

By conditions $L x=-f(t), L x_{n}=0, \sum_{\tau=a+1}^{b+1} f(\tau) x_{n}(\tau)=0$, and (9), observe that

$$
\sum_{\tau=a+1}^{b+1} f(\tau) x_{n}(\tau)=\left\{p(t) \omega\left[x(t), x_{n}(t)\right]\right\}_{a}^{b+1}=0 ;
$$

then

$$
\begin{gathered}
p(b+1)\left[x(b+1) \Delta x_{n}(b+1)-x_{n}(b+1) \Delta x(b+1)\right] \\
=p(a)\left[x(a) \Delta x_{n}(a)-x_{n}(a) \Delta x(a)\right]
\end{gathered}
$$

that is, $U_{1}(x)=\alpha_{1} x(a)+\alpha_{2} \Delta x(a)=0$. Thus, $x(t)$ is a solution of problem (9), and this completes the proof.

In what follows, we begin to build the generalized Green function.

Theorem 7. If the S-L problem (3) has a solution $x_{0}(t)$, and $\sum_{t=a+1}^{b+1} x_{0}^{2}(t)=1$, then there exists a unique function $G(t, \tau)$ which satisfies the following properties:

(1) $L G(t, \tau)=x_{0}(t) x_{0}(\tau), U_{1}(G)=0, t \in[a, \tau]$;

(2) $L G(t, \tau)=x_{0}(t) x_{0}(\tau), U_{2}(G)=0, t \in[\tau, b+1]$;

(3) $\left.\Delta_{t} G(t, \tau)\right|_{t=\tau+0}-\left.\Delta_{t} G(t, \tau)\right|_{t=\tau-0}=-(1 / p(\tau))$;

(4) $\sum_{t=a+1}^{b+1} G(t, \tau) x_{0}(t)=0$.

The function having the above properties is called the generalized Green function of the problem (1).

Proof. Choose another solution $x_{1}(t)$ of $L x=0$ which is linearly independent with $x_{0}(t)$, such that

$$
p(t)\left[x_{0}(t) \Delta x_{1}(t)-x_{1}(t) \Delta x_{0}(t)\right]=1 .
$$

Let $\omega(t)$ be any one particular solution of $L x=x_{0}(t) x_{0}(\tau)$. Thus, by the structure of general solution for nonhomogeneous difference equations, we know that

$$
G(t, \tau)= \begin{cases}\omega(t)+c_{1} x_{0}(t)+c_{2} x_{1}(t), & t \in[a, \tau], \\ \omega(t)+c_{3} x_{0}(t)+c_{4} x_{1}(t), & t \in[\tau, b+1],\end{cases}
$$

where $c_{i}(i=1,2,3,4)$ can be contained by the properties of $G(t, \tau)$.

Firstly, let $G(t, \tau)$ satisfy the boundary conditions

$$
\begin{aligned}
& U_{1}(G)=U_{1}(\omega)+c_{1} U_{1}\left(x_{0}\right)+c_{2} U_{1}\left(x_{1}\right)=0, \\
& U_{2}(G)=U_{2}(\omega)+c_{3} U_{2}\left(x_{0}\right)+c_{4} U_{2}\left(x_{1}\right)=0 .
\end{aligned}
$$

By the fact that $U_{1}\left(x_{0}\right)=0$ and $U_{2}\left(x_{0}\right)=0$, we get that

$$
\begin{aligned}
& U_{1}(G)=U_{1}(\omega)+c_{2} U_{1}\left(x_{1}\right)=0, \\
& U_{2}(G)=U_{2}(\omega)+c_{4} U_{2}\left(x_{1}\right)=0 .
\end{aligned}
$$


Since $x_{0}(t)$ and $x_{1}(t)$ are linearly independent, thus, $U_{1}\left(x_{1}\right) \neq$ 0 and $U_{2}\left(x_{1}\right) \neq 0$, so we get that $c_{2}$ and $c_{4}$ are

$$
c_{2}=-\frac{U_{1}(\omega)}{U_{1}\left(x_{1}\right)}, \quad c_{4}=-\frac{U_{2}(\omega)}{U_{2}\left(x_{1}\right)}
$$

then

$$
c_{2}-c_{4}=\frac{U_{2}(\omega)}{U_{2}\left(x_{1}\right)}-\frac{U_{1}(\omega)}{U_{1}\left(x_{1}\right)} .
$$

However, from (16) and $\left.\Delta_{t} G(t, \tau)\right|_{t=\tau+0}-\left.\Delta_{t} G(t, \tau)\right|_{t=\tau-0}=$ $-(1 / p(\tau))$ we get

$$
\begin{gathered}
\left(c_{1}-c_{3}\right) x_{0}(\tau)+\left(c_{2}-c_{4}\right) x_{1}(\tau)=0, \\
\left(c_{1}-c_{3}\right) \Delta x_{0}(\tau)+\left(c_{2}-c_{4}\right) \Delta x_{1}(\tau)=\frac{1}{p(\tau)} .
\end{gathered}
$$

By (21), we get

$$
c_{1}-c_{3}=-x_{1}(\tau), \quad c_{2}-c_{4}=x_{0}(\tau) .
$$

In the following we will prove that (20) is consistent with (22).

By

$$
\begin{gathered}
p(a)\left[x_{0}(a) \Delta x_{1}(a)-x_{1}(a) \Delta x_{0}(a)\right]=1, \\
p(b+1)\left[x_{0}(b+1) \Delta x_{1}(b+1)\right. \\
\left.-x_{1}(b+1) \Delta x_{0}(b+1)\right]=1, \\
\alpha_{1} x_{0}(a)+\alpha_{2} \Delta x_{0}(a)=0 \\
\beta_{1} x_{0}(b+1)+\beta_{2} \Delta x_{0}(b+1)=0,
\end{gathered}
$$

we get

$$
\begin{gathered}
x_{0}(a)=\frac{\alpha_{2}}{p(a) U_{1}\left(x_{1}\right)}, \\
\Delta x_{0}(a)=\frac{-\alpha_{1}}{p(a) U_{1}\left(x_{1}\right)}, \\
x_{0}(b+1)=\frac{\beta_{2}}{p(b+1) U_{2}\left(x_{1}\right)}, \\
\Delta x_{0}(b+1)=\frac{-\beta_{1}}{p(b+1) U_{2}\left(x_{1}\right)} .
\end{gathered}
$$

From

$$
x_{0} L \omega-\omega L x_{0}=\Delta\left[p\left(x_{0} \Delta \omega-\omega \Delta x_{0}\right)\right](t-1)
$$

and $\sum_{t=a+1}^{b+1} x_{0}^{2}(t)=1$ we get

$$
\begin{aligned}
x_{0}(\tau)= & {\left[p\left(x_{0} \Delta \omega-\omega \Delta x_{0}\right)\right]_{a}^{b+1} } \\
= & p(b+1)\left[x_{0}(b+1) \Delta \omega(b+1)\right. \\
& \left.\quad-\omega(b+1) \Delta x_{0}(b+1)\right] \\
& -p(a)\left[x_{0}(a) \Delta \omega(a)-\omega(a) \Delta x_{0}(a)\right] .
\end{aligned}
$$

Now, we get

$$
x_{0}(\tau)=\frac{U_{2}(\omega)}{U_{2}\left(x_{1}\right)}-\frac{U_{1}(\omega)}{U_{1}\left(x_{1}\right)}=c_{2}-c_{4} ;
$$

that is, (20) and (22) are consistent.

In order to determine $c_{1}$ and $c_{3}$, by $c_{1}-c_{3}=-x_{1}(\tau)$, we obtain $c_{1}=c_{3}-x_{1}(\tau)$; putting $c_{1}$ into $\sum_{t=a+1}^{b+1} G(t, \tau) x_{0}(t)=0$, we get

$$
\begin{gathered}
\sum_{t=a+1}^{b+1} \omega(t) x_{0}(t)+c_{3} \sum_{t=a+1}^{\tau-1} x_{0}^{2}(t)-x_{1}(\tau) \sum_{t=a+1}^{\tau-1} x_{0}^{2}(t) \\
-\frac{U_{1}(\omega)}{U_{1}\left(x_{1}\right)} \sum_{t=a+1}^{\tau-1} x_{0}(t) x_{1}(t)+c_{3} \sum_{t=\tau}^{b+1} x_{0}^{2}(t) \\
-\frac{U_{2}(\omega)}{U_{2}\left(x_{1}\right)} \sum_{t=\tau}^{b+1} x_{0}(t) x_{1}(t)=0 ;
\end{gathered}
$$

then

$$
\begin{aligned}
c_{3}= & -\sum_{t=a+1}^{b+1} \omega(t) x_{0}(t)+x_{1}(\tau) \sum_{t=a+1}^{\tau-1} x_{0}^{2}(t) \\
& +\frac{U_{1}(\omega)}{U_{1}\left(x_{1}\right)} \sum_{t=a+1}^{\tau-1} x_{0}(t) x_{1}(t) \\
& +\frac{U_{2}(\omega)}{U_{2}\left(x_{1}\right)} \sum_{t=\tau}^{b+1} x_{0}(t) x_{1}(t) .
\end{aligned}
$$

All these show that we can get $c_{1}, c_{2}, c_{3}$, and $c_{4}$ uniquely one by one; then $G(t, \tau)$ is unique too; that is, the function $G(t, \tau)$ constructed by the above steps has all the properties of generalized Green's function. This completes the proof.

Example 8. Consider the generalized Green function of the $\mathrm{S}$-L problem

$$
\begin{aligned}
& \Delta^{2} x(t-1)+2 x(t)=0, \\
& x(0)=0, \quad x(2)=0 .
\end{aligned}
$$

Proof. From the conditions $\sum_{t=1}^{2} x_{0}^{2}(t)=1$ and $p(t)\left[x_{0}(t) \Delta x_{1}(t)-x_{1}(t) \Delta x_{0}(t)\right]=1$, we can get

$$
x_{0}(t)=\sin \frac{\pi}{2} t, \quad x_{1}(t)=-\cos \frac{\pi}{2} t .
$$

It is easy to verify that

$$
\omega(t)=\frac{1}{4} \sin \frac{\pi}{2} \tau \sin \frac{\pi}{2} t-\frac{t}{2} \sin \frac{\pi}{2} \tau \cos \frac{\pi}{2} t
$$

is a particular solution of the following equation:

$$
\Delta^{2} \omega(t-1)+2 \omega(t)=\sin \frac{\pi}{2} \tau \sin \frac{\pi}{2} t .
$$

Note that

$$
\begin{gathered}
U_{1}\left(x_{1}\right)=x_{1}(0)=-1, \quad U_{1}(\omega)=\omega(0)=0, \\
U_{2}\left(x_{1}\right)=x_{1}(2)=1, \quad U_{2}(\omega)=\omega(2)=\sin \frac{\pi}{2} \tau .
\end{gathered}
$$


So

$$
\begin{gathered}
c_{2}=0, \quad c_{4}=-\sin \frac{\pi}{2} \tau \\
c_{3}=-\frac{1}{2}\left(\tau \cos \frac{\pi}{2} \tau+\frac{1}{2} \sin \frac{\pi}{2} \tau\right)-\frac{1}{4} \cos \frac{\pi}{2} \tau \\
c_{1}=c_{3}-x_{1}(\tau)=-\frac{1}{2}\left(\tau \cos \frac{\pi}{2} \tau+\frac{1}{2} \sin \frac{\pi}{2} \tau\right)+\frac{3}{4} \cos \frac{\pi}{2} \tau
\end{gathered}
$$

then we can get the generalized Green function

$$
G(t, \tau)=\left\{\begin{aligned}
-\frac{t}{2} & \sin \frac{\pi}{2} \tau \cos \frac{\pi}{2} t \\
& +\frac{3-2 \tau}{4} \sin \frac{\pi}{2} t \cos \frac{\pi}{2} \tau, \quad t \in[0, \tau], \\
\frac{2-t}{2} & \sin \frac{\pi}{2} \tau \cos \frac{\pi}{2} t \\
& -\frac{2 \tau+1}{4} \sin \frac{\pi}{2} t \cos \frac{\pi}{2} \tau, \quad t \in[\tau, 2] .
\end{aligned}\right.
$$

Now, we prove that by the extended Green function it still can convert the originally irreversible difference operator into reversible form, and its summation form is unique.

Theorem 9. Let $G(t, \tau)$ be the generalized Green function of the S-L problem (2), and $x_{0}(t)$ satisfying $\sum_{t=a+1}^{b+1} x_{0}^{2}(t)=1$ is a solution of the $S-L$ problem, and $\sum_{\tau=a+1}^{b+1} f(\tau) x_{0}(\tau)=0$. Then the solution of the semihomogeneous boundary value problem

$$
L x=-f(t), \quad U_{1}(x)=0, \quad U_{2}(x)=0
$$

can be expressed by

$$
x(t)=\sum_{\tau=a+1}^{b+1} G(t, \tau) f(\tau) .
$$

Proof. By the fact that

$$
\begin{gathered}
\Delta x(t-1)=\sum_{\tau=a+1}^{b+1} \Delta G(t-1, \tau) f(\tau), \\
\Delta^{2} x(t-1)=\Delta\left[\sum_{\tau=a+1}^{t-1} \Delta G(t-1, \tau) f(\tau)\right. \\
\left.+\sum_{\tau=t}^{b+1} \Delta G(t-1, \tau) f(\tau)\right] \\
=\left\{\sum_{\tau=a+1}^{t-1}[G(t, \tau) f(\tau)-G(t-1, \tau) f(\tau)]\right. \\
\left.\quad+\sum_{\tau=t}^{b+1}[G(t, \tau) f(\tau)-G(t-1, \tau) f(\tau)]\right\}
\end{gathered}
$$

$$
\begin{aligned}
=\Delta[G(t, t-1) f(t-1) & \\
& \quad-G(t-1, a+1) f(a+1) \\
& \quad+G(t, b+1) f(b+1)-G(t-1, t) f(t)] \\
= & {[\Delta G(t, b+1) f(b+1)} \\
& \quad-\Delta G(t-1, a+1) f(a+1)] \\
+ & {[\Delta G(t, t-1) f(t-1)-\Delta G(t-1, t) f(t)] } \\
=\sum_{\tau=a+1}^{b+1} \Delta^{2} G(t-1, \tau) f(\tau) & +\left[\left.\Delta_{t} G(\tau, t)\right|_{\tau=t+0}-\left.\Delta_{t} G(\tau, t)\right|_{\tau=t-0}\right] f(t) \\
= & \sum_{\tau=a+1}^{b+1} \Delta^{2} G(t-1, \tau) f(\tau)-\frac{f(t)}{p(t)},
\end{aligned}
$$

then

$$
\begin{aligned}
L x= & \Delta p(t-1) \Delta x(t-1)+p(t) \Delta^{2} x(t-1) \\
& +q(t) x(t)+\lambda r(t) x(t) \\
= & \sum_{\tau=a+1}^{b+1}(L G) f(\tau)-f(t) \\
= & \sum_{\tau=a+1}^{b+1} x_{0}(t) x_{0}(\tau) f(\tau)-f(t)=-f(t) .
\end{aligned}
$$

To prove the uniqueness, it needs to be proved that we cannot find $\tilde{x}(t)$ and $f(t)$ which satisfy $\sum_{\tau=a+1}^{b+1} f(\tau) x_{0}(\tau)=0$ except $x_{0}(t)$ and $f(t)$; if there exists $\tilde{x}(t)$, then by Theorem 6 , we know that $\tilde{x}(t)$ is also an eigenfunction of the S-L problem; this shows that $\tilde{x}(t)$ and $x_{0}(t)$ are linearly dependent, which completes the proof.

\section{The Main Results for Problem (2)}

In what follows, we build the generalized Green function of the periodic S-L problem.

Theorem 10. If the periodic $S$ - $L$ problem (2) has a solution $x_{0}(t)$, and choosing a linearly independent solution $x_{1}(t)$ with $x_{0}(t)$ for $L x=0$, then there exists a function $G(t, \tau)$ which satisfies the following properties:

(1) $L G(t, \tau)=x_{0}(t) x_{0}(\tau), U_{3}(G)=0, t \in[a, \tau]$;

(2) $L G(t, \tau)=x_{0}(t) x_{0}(\tau), U_{4}(G)=0, t \in[\tau, b+1]$;

(3) $\left.\Delta_{t} G(t, \tau)\right|_{t=\tau+0}-\left.\Delta_{t} G(t, \tau)\right|_{t=\tau-0}=-(1 / p(\tau))$;

(4) $\sum_{t=a+1}^{b+1} G(t, \tau) x_{0}(t)=0$ and $\sum_{t=a+1}^{b+1} G(t, \tau) x_{1}(t)=0$.

The function having the above properties is called the generalized Green function to the problem (2). 
Proof. Choose $x_{1}(t)$ so that

$$
p(t)\left[x_{0}(t) \Delta x_{1}(t)-x_{1}(t) \Delta x_{0}(t)\right]=1 \text {. }
$$
then

Let $\omega(t)$ be any one particular solution to $L x=x_{0}(t) x_{0}(\tau)$;

$$
G(t, \tau)= \begin{cases}\omega(t)+c_{1} x_{0}(t)+c_{2} x_{1}(t), & t \in[a, \tau], \\ \omega(t)+c_{3} x_{0}(t)+c_{4} x_{1}(t), & t \in[\tau, b+1],\end{cases}
$$

where $c_{i}(i=1,2,3,4)$ can be contained by the properties of $G(t, \tau)$.

Firstly, let $G(t, \tau)$ satisfy the boundary conditions

$$
\begin{aligned}
& U_{3}(G)=U_{3}(\omega)+c_{1} U_{3}\left(x_{0}\right)+c_{2} U_{3}\left(x_{1}\right)=0, \\
& U_{4}(G)=U_{4}(\omega)+c_{3} U_{4}\left(x_{0}\right)+c_{4} U_{4}\left(x_{1}\right)=0 .
\end{aligned}
$$

By the fact that $U_{3}\left(x_{0}\right)=0, U_{4}\left(x_{0}\right)=0$ and in the periodic problem we have $U_{3}\left(x_{1}\right)=U_{4}\left(x_{1}\right)=0$; then $U_{3}(\omega)=U_{4}(\omega)=$ 0 .

Thus, $c_{i}$ can be contained; that is, by the conditions (3) and (4), we get

$$
c_{1}-c_{3}=-x_{1}(\tau), \quad c_{2}-c_{4}=x_{0}(\tau) .
$$

In order to determine $c_{i}$, by $c_{1}-c_{3}=-x_{1}(\tau)$, we have $c_{1}=$ $c_{3}-x_{1}(\tau)$; by $c_{2}-c_{4}=x_{0}(\tau)$, we have $c_{2}=c_{4}+x_{0}(\tau)$; putting $c_{1}, c_{2}$ into $\sum_{t=a+1}^{b+1} G(t, \tau) x_{0}(t)=0$ and $\sum_{t=a+1}^{b+1} G(t, \tau) x_{1}(t)=0$ respectively, it shows that

$$
\begin{gathered}
c_{3} \sum_{t=a+1}^{b+1} x_{0}^{2}(t)+c_{4} \sum_{t=a+1}^{b+1} x_{0}(t) x_{1}(t) \\
=-\sum_{t=a+1}^{b+1} \omega(t) x_{0}(t)+x_{1}(\tau) \sum_{t=a+1}^{\tau-1} x_{0}^{2}(t) \\
\quad-x_{0}(\tau) \sum_{t=a+1}^{\tau-1} x_{0}(t) x_{1}(t), \\
c_{3} \sum_{t=a+1}^{b+1} x_{0}(t) x_{1}(t)+c_{4} \sum_{t=a+1}^{b+1} x_{1}^{2}(t) \\
=-\sum_{t=a+1}^{b+1} \omega(t) x_{1}(t)+x_{1}(\tau) \sum_{t=a+1}^{\tau-1} x_{0}(t) x_{1}(t) \\
\quad-x_{0}(\tau) \sum_{t=a+1}^{\tau-1} x_{1}^{2}(t) .
\end{gathered}
$$

Combine the above equations; $c_{3}$ and $c_{4}$ can be determined; then $c_{1}$ and $c_{2}$ can be determined, so $G(t, \tau)$ also can be determined; that is, the function $G(t, \tau)$ set by the above steps has all the properties of generalized Green's function. This completes the proof.

Remark 11. Because the choice of $x_{0}(t)$ in the above structure of generalized Green's function is not unique, so the generalized Green function of periodic S-L problem (2) is changed with $x_{0}(t)$, to have different form.

Example 12. Consider the generalized Green function of the periodic S-L problem

$$
\begin{gathered}
\Delta^{2} x(t-1)+2 x(t)=0, \\
x(0)=x(4), \quad \Delta x(0)=\Delta x(4) .
\end{gathered}
$$

Proof. Let $x_{0}(t)=\sin (\pi / 2) t$, and from

$$
p(t)\left[x_{0}(t) \Delta x_{1}(t)-x_{1}(t) \Delta x_{0}(t)\right]=1,
$$

we can let $x_{1}(t)=-\cos (\pi / 2) t$, (where $\left.p(t)=1\right)$. It is easy to verify that

$$
\omega(t)=\frac{1}{4} \sin \frac{\pi}{2} \tau \sin \frac{\pi}{2} t-\frac{t}{2} \sin \frac{\pi}{2} \tau \cos \frac{\pi}{2} t
$$

is a particular solution of the following equation:

$$
\Delta^{2} \omega(t-1)+2 \omega(t)=\sin \frac{\pi}{2} \tau \sin \frac{\pi}{2} t
$$

By Theorem 10, we have

$$
\begin{aligned}
c_{3}= & -\frac{1}{4} \sin \frac{\pi}{2} \tau-\frac{1}{2} \cos \frac{\pi}{2} \tau\left(\frac{\tau}{2}+\frac{1}{4} \cos \pi \tau-\frac{1}{4}\right) \\
& -\frac{1}{8} \sin \frac{\pi}{2} \tau \sin \pi \tau, \\
c_{4}=-\frac{1}{8} & \cos \frac{\pi}{2} \tau \sin \pi \tau-\frac{1}{2} \sin \frac{\pi}{2} \tau\left(-\frac{1}{4} \cos \pi \tau+\frac{\tau}{2}+\frac{9}{4}\right)
\end{aligned}
$$

then

$$
\begin{aligned}
c_{1}= & -\frac{1}{4} \sin \frac{\pi}{2} \tau-\frac{1}{2} \cos \frac{\pi}{2} \tau\left(\frac{\tau}{2}-\frac{9}{4}+\frac{1}{4} \cos \pi \tau\right) \\
& -\frac{1}{8} \sin \frac{\pi}{2} \tau \sin \pi \tau, \\
c_{2}= & -\frac{1}{8} \cos \frac{\pi}{2} \tau \sin \pi \tau-\frac{1}{2} \sin \frac{\pi}{2} \tau\left(-\frac{1}{4} \cos \pi \tau+\frac{\tau}{2}+\frac{1}{4}\right) .
\end{aligned}
$$


So we can get the generalized Green function

$$
G(t, \tau)=\left\{\begin{array}{rl}
-\frac{1}{2} \sin \frac{\pi}{2} t \cos \frac{\pi}{2} \tau\left(\frac{\tau}{2}+\frac{1}{4} \cos \pi \tau-\frac{9}{4}\right)-\frac{1}{8} \sin \frac{\pi}{2} \tau \sin \pi \tau \sin \frac{\pi}{2} t & \\
& +\frac{1}{8} \cos \frac{\pi}{2} t \cos \frac{\pi}{2} \tau \sin \pi \tau \\
& +\frac{1}{2} \sin \frac{\pi}{2} \tau \cos \frac{\pi}{2} t\left(-\frac{1}{4} \cos \pi \tau+\frac{\tau}{2}+\frac{1}{4}-t\right), \\
-\frac{1}{2} \sin \frac{\pi}{2} t \cos \frac{\pi}{2} \tau\left(\frac{\tau}{2}-\frac{1}{4}+\frac{1}{4} \cos \pi \tau\right)-\frac{1}{8} \sin \frac{\pi}{2} \tau \sin \pi \tau \sin \frac{\pi}{2} t & t \in[0, \tau], \\
& +\frac{1}{8} \cos \frac{\pi}{2} t \cos \frac{\pi}{2} \tau \sin \pi \tau \\
& +\frac{1}{2} \sin \frac{\pi}{2} \tau \cos \frac{\pi}{2} t\left(-\frac{1}{4} \cos \pi \tau+\frac{\tau}{2}+\frac{9}{4}-t\right),
\end{array} \quad t \in[\tau, 4] .\right.
$$

However, if we put $x_{0}(t)=-\cos (\pi / 2) t$, then the generalized Green function is

$$
G(t, \tau)=\left\{\begin{array}{rl}
\frac{1}{2} \sin \frac{\pi}{2} t \cos \frac{\pi}{2} \tau\left(\frac{1}{4} \cos \pi \tau-\frac{17-t-2 \tau}{4}\right)-\frac{1}{8} \cos \frac{\pi}{2} \tau \sin \pi \tau \cos \frac{\pi}{2} t & \\
& +\frac{1}{8} \sin \frac{\pi}{2} t \sin \frac{\pi}{2} \tau \sin \pi \tau \\
& +\frac{1}{4} \cos \frac{\pi}{2} \tau \cos \frac{\pi}{2} t\left(-\frac{1}{4} \cos \pi \tau+\frac{\tau}{2}-\frac{15}{4}\right), \\
\frac{1}{2} \sin \frac{\pi}{2} t \cos \frac{\pi}{2} \tau\left(\frac{2 \tau-9+4 t}{4}+\frac{1}{4} \cos \pi \tau\right)-\frac{1}{8} \cos \frac{\pi}{2} \tau \sin \pi \tau \cos \frac{\pi}{2} t & t \in[0, \tau], \\
& +\frac{1}{8} \sin \frac{\pi}{2} t \sin \frac{\pi}{2} \tau \sin \pi \tau \\
& -\frac{1}{2} \sin \frac{\pi}{2} \tau \cos \frac{\pi}{2} t\left(-\frac{1}{4} \cos \pi \tau+\frac{\tau}{2}-\frac{3}{4}\right),
\end{array} \quad t \in[\tau, 4] .\right.
$$

Be similar to Theorem 9, we can get the following result; however, because the generalized Green function of the periodic S-L problem (2) is not unique, summation form of difference operator is also not unique.

Theorem 13. Let $G(t, \tau)$ be the generalized Green function of the periodic $S$-L problem (2); then the solution of the semihomogeneous boundary value problem

$$
L x=-f(t), \quad U_{3}(x)=0, \quad U_{4}(x)=0
$$

can be expressed by

$$
x(t)=\sum_{\tau=a+1}^{b+1} G(t, \tau) f(\tau) .
$$

\section{Conflict of Interests}

The authors declare that there is no conflict of interests regarding the publication of this paper.

\section{Acknowledgments}

The authors are grateful to the anonymous referees for their valuable suggestions. This research was supported by the National Natural Science Foundation of China (11101335 and 11201378).

\section{References}

[1] D. Bai and Y. Xu, "Nontrivial solutions of boundary value problems of second-order difference equations," Journal of Mathematical Analysis and Applications, vol. 326, no. 1, pp. 297302, 2007. 
[2] G. Zhang and Z. Yang, "Positive solutions of a general discrete boundary value problem," Journal of Mathematical Analysis and Applications, vol. 339, no. 1, pp. 469-481, 2008.

[3] R. Ma, Y. Lu, and T. Chen, "Existence of one-signed solutions of discrete second-order periodic boundary value problems," Abstract and Applied Analysis, vol. 2012, Article ID 437912, 13 pages, 2012.

[4] C. Gao, "Necessary and sufficient condition for the existence of solutions to a discrete second-order boundary value problem," Abstract and Applied Analysis, vol. 2012, Article ID 951251, 12 pages, 2012.

[5] W. G. Kelley and A. C. Peterson, Difference Equations: An Introduction with Applications, Academic Press, New York, NY, USA, 2001.

[6] C. S. Goodrich, "Continuity of solutions to discrete fractional initial value problems," Computers \& Mathematics with Applications, vol. 59, no. 11, pp. 3489-3499, 2010.

[7] C. S. Goodrich, "Solutions to a discrete right-focal fractional boundary value problem," International Journal of Difference Equations, vol. 5, no. 2, pp. 195-216, 2010.

[8] C. S. Goodrich, "On a discrete fractional three-point boundary value problem," Journal of Difference Equations and Applications, vol. 18, no. 3, pp. 397-415, 2010.

[9] R. Agarwal and P. Y. Wong, Advanced Topics in Difference Equations, Springer, New York, NY, USA, 1997.

[10] C. Gao and J. Xu, "Bifurcation techniques and positive solutions of discrete Lidstone boundary value problems," Applied Mathematics and Computation, vol. 218, no. 2, pp. 434-444, 2011. 


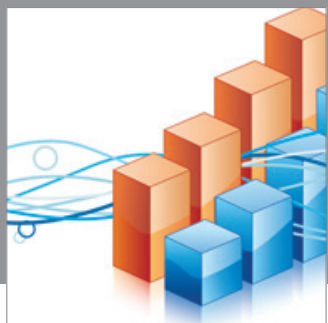

Advances in

Operations Research

mansans

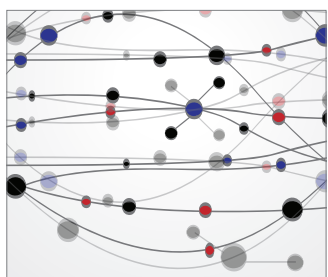

The Scientific World Journal
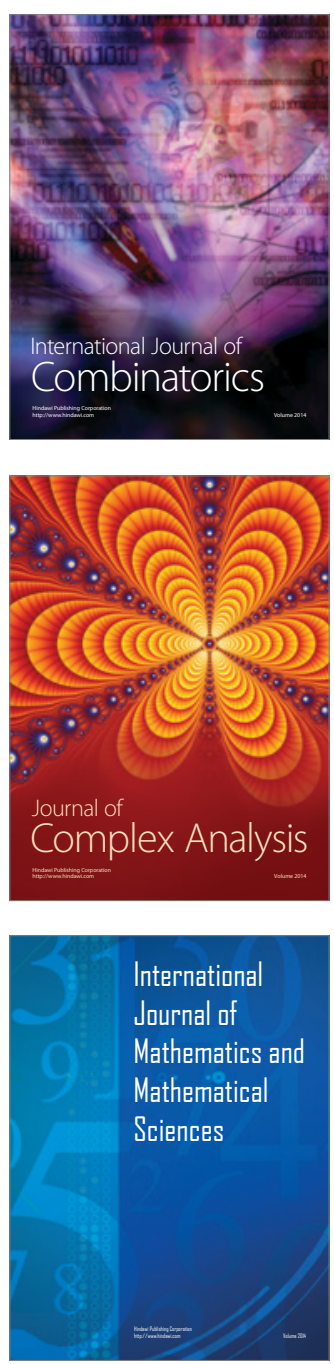
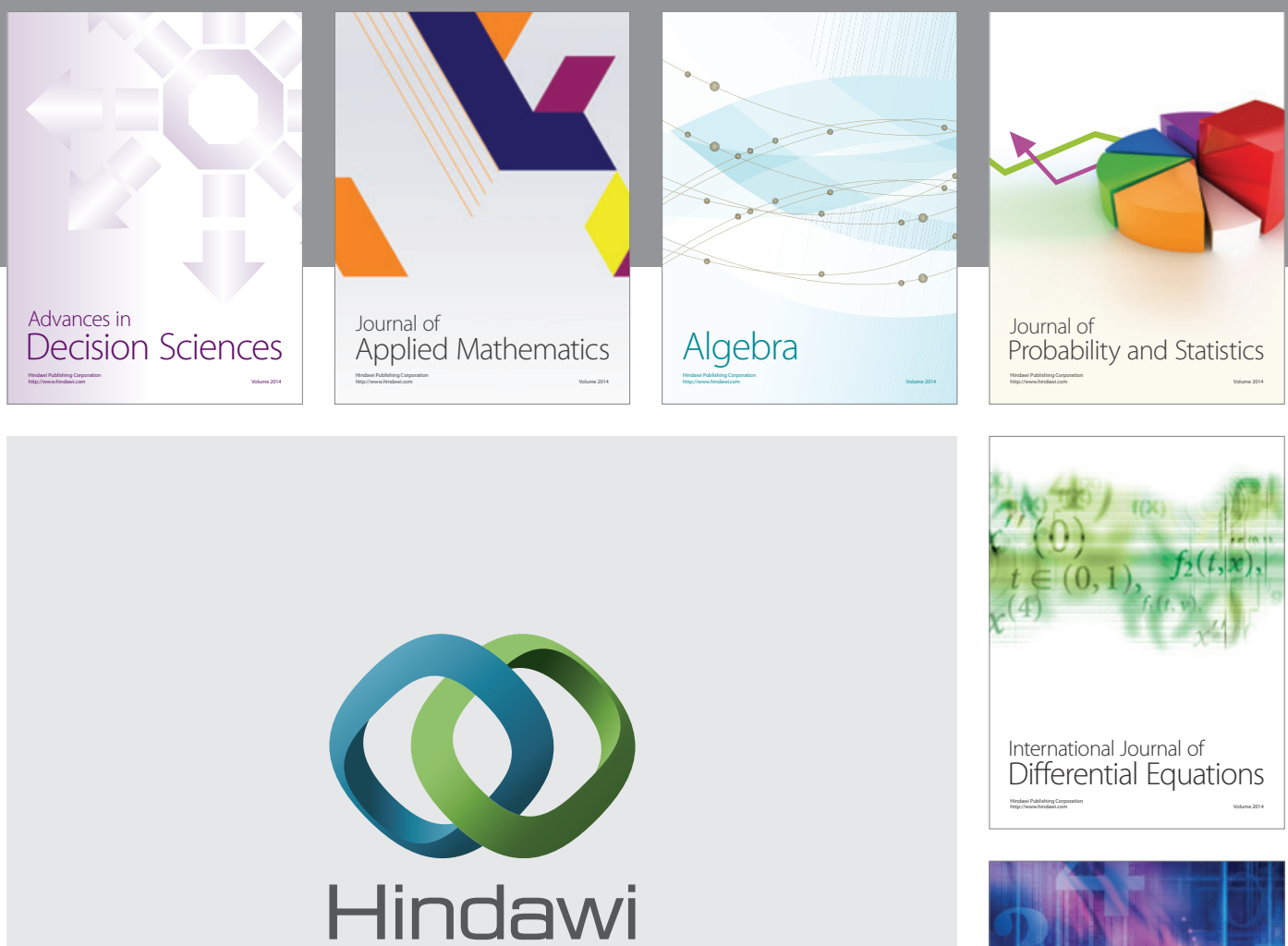

Submit your manuscripts at http://www.hindawi.com
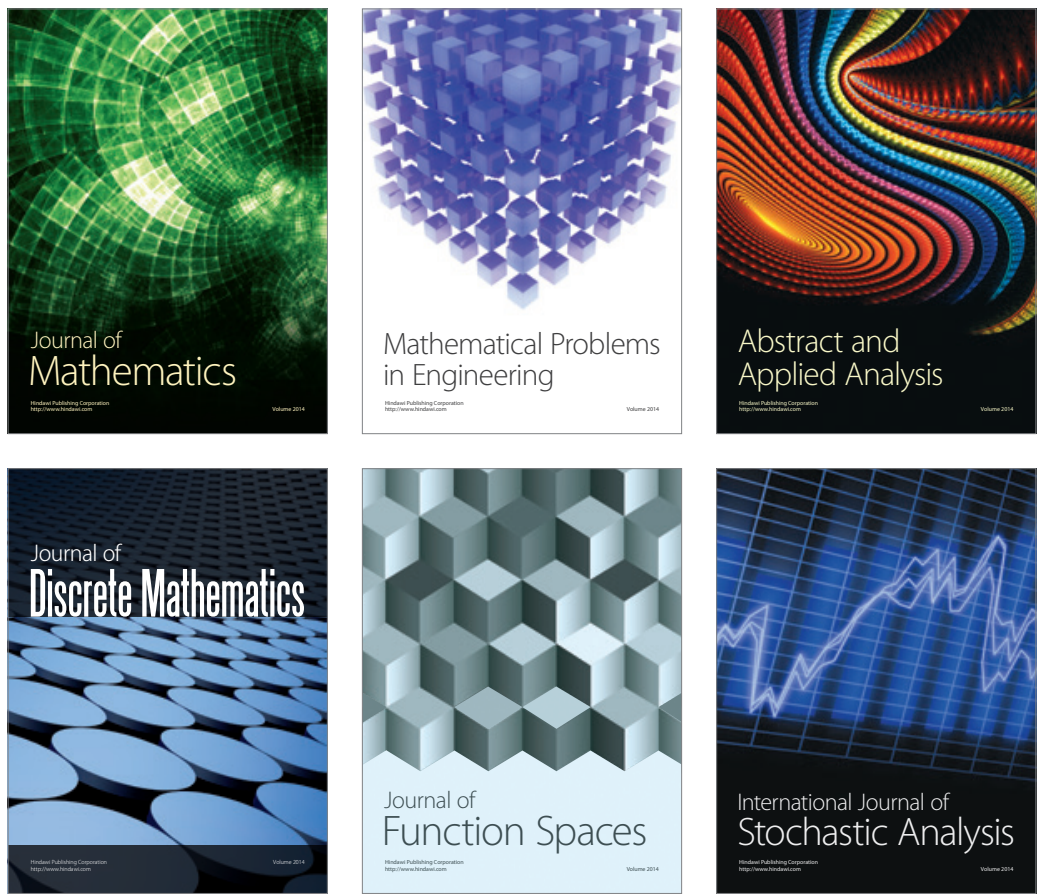

Journal of

Function Spaces

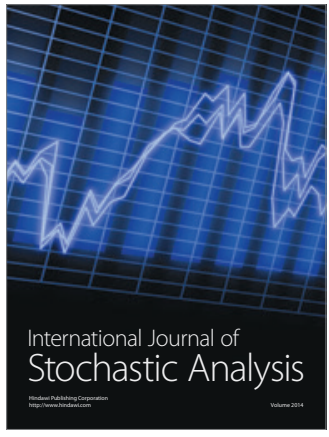

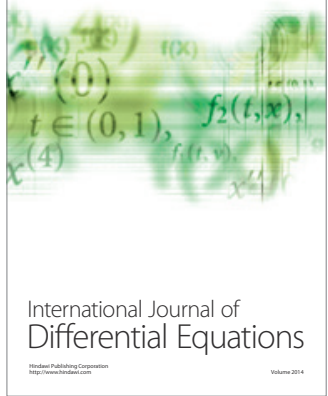
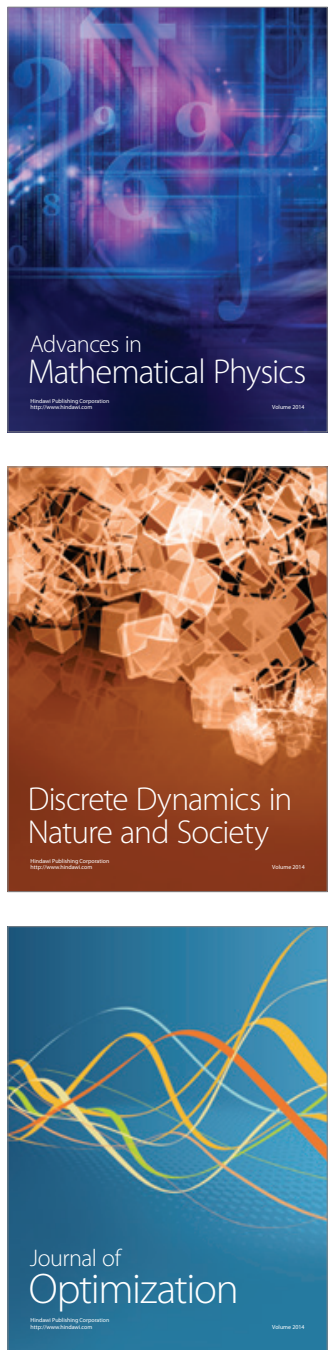